\title{
Accelerator Measurements of the Askaryan effect in Rock Salt: A Roadmap Toward Teraton Underground Neutrino Detectors
}

\author{
P. W. Gorham, ${ }^{1}$ D. Saltzberg, ${ }^{2}$ R. C. Field, ${ }^{3}$ E. Guillian, ${ }^{1}$ R. Milincic, ${ }^{1}$ D. Walz,${ }^{3}$ and D. Williams ${ }^{2}$ \\ ${ }^{1}$ Dept. of Physics \& Astronomy, Univ. of Hawaii at Manoa, 2505 Correa Rd. Honolulu, HI, 96822 \\ ${ }^{2}$ Dept. of Physics \& Astronomy, Univ. of Calif. at Los Angeles, Los Angeles, CA \\ ${ }^{3}$ Stanford Linear Accelerator Center, Stanford University, Menlo Park California
}

\begin{abstract}
We report on further SLAC measurements of the Askaryan effect: coherent radio emission from charge asymmetry in electromagnetic cascades. We used synthetic rock salt as the dielectric medium, with cascades produced by $\mathrm{GeV}$ bremsstrahlung photons at the Final Focus Test Beam. We extend our prior discovery measurements to a wider range of parameter space and explore the effect in a dielectric medium of great potential interest to large scale ultra-high energy neutrino detectors: rock salt (halite), which occurs naturally in high purity formations containing in many cases hundreds of $\mathrm{km}^{3}$ of water-equivalent mass. We observed strong coherent pulsed radio emission over a frequency band from 0.2-15 GHz. A grid of embedded dual-polarization antennas was used to confirm the high degree of linear polarization and track the change of direction of the electric-field vector with azimuth around the shower. Coherence was observed over 4 orders of magnitude of shower energy. The frequency dependence of the radiation was tested over two orders of magnitude of UHF and microwave frequencies. We have also made the first observations of coherent transition radiation from the Askaryan charge excess, and the result agrees well with theoretical predictions. Based on these results we have performed detailed and conservative simulation of a realistic GZK neutrino telescope array within a salt-dome, and we find it capable of detecting 10 or more contained events per year from even the most conservative GZK neutrino models.
\end{abstract}

\section{INTRODUCTION}

It is now widely understood that the universe becomes largely opaque to photons above about $100 \mathrm{TeV}$ due to pair production on the cosmic infrared and microwave background [1]. Stable charged baryons or leptons do not become magnetically rigid enough to propagate over intergalactic distances until their energies are so high that they also suffer significant losses through interactions with the cosmic microwave background, through the process first noted by Greisen, and Zatsepin \& Kuzmin (GZK) in the early 1960's [2, 3].

Extragalactic astronomy in the $10^{15-21} \mathrm{eV}$ energy range at $\geq 0.1 \mathrm{Gpc}$ scales must therefore utilize other messengers, and neutrinos are the most likely contender. Moreover, the existence of sources that can produce single particles with energies approaching 1 Zeta-electron-volt $\left(10^{21} \mathrm{eV}=1 \mathrm{ZeV}=60\right.$ Joules) provides a compelling driver toward development of ultra-high energy neutrino detectors, which can shed light not only on acceleration processes at the sources that produce the primary cosmic rays, but on the acute problem of their propagation and attenuation in the intergalactic medium through the GZK process. This process itself yields neutrinos as secondaries of the $p \gamma_{2.7 K}$ interactions, and the resulting GZK neutrino spectrum [4], peaking at $\mathrm{EeV}$ energies, distinctly reflects the cosmic ray source spectrum and distribution and provides unique diagnostics on the evolutionary history of the cosmic ray accelerators. Because the GZK neutrino flux arises from the aggregate intensity of all cosmic ray sources at all epochs, it is also potentially the strongest source of $\mathrm{EeV}$ neutrinos integrated over the entire sky, and may in fact act as a "standard candle" for EeV neutrinos. Also, given the $\sim 100 \mathrm{TeV}$ center-of-momentum energy of GZK neutrino interactions on nucleons at Earth, the possibility of high-energy physics applications cannot be ignored. The combination of wide accep- tance for the flux models with a strong science motivation has made the detection of GZK neutrinos the focus of significant attention for all current high energy neutrino detectors.

This goal compels us to consider detectors with target masses approaching $10^{42}$ nucleons, a Teraton of mass, or 1000 $\mathrm{km}^{3}$ water equivalent at EeV energies [5]. Such a target mass must be physically accessible, and must be able to transmit information about embedded neutrino cascades within its enormous volume to a suitable and cost-effective detector array. A promising approach toward this daunting task is to utilize the Askaryan effect [6, 7], a process which leads to strong, coherent radio pulses from such cascades. Several known dielectric media, with ice and rock salt the most promising at present, appear to have the necessary characteristics. Antarctic ice has been the focus of studies for this goal for several years now via the Radio Ice Cerenkov Experiment (RICE) [8], and the Antarctic Impulsive Transient Antenna (ANITA) longduration balloon project will seek to exploit the effect in a novel approach [9]. A recent limit at extremely high neutrino energies has been reported via FORTE satellite observations of the Greenland ice sheet [10]. There is also another class of experiments searching for Čerenkov emission from UHE neutrinos via neutrino interactions with lunar regolith [11-14].

Rock salt, first suggested as a possible target medium by Askaryan [6], has also been the focus of recent efforts, and it appears to show equal promise with ice in regard to potential for detector development, along with the likely advantage of greater accessibility $[15,16]$. We report here on experiments to further understand aspects of the Askaryan process with a particular focus on rock salt as the medium for the cascades and radio pulse production. Our results now extend measurements of the effect over the entire range of cascade energies and radio frequency range of interest for GZK neutrino detection. We have also explored in greater detail the shower calorimetry aspects of radio Čerenkov measurements, 
and have made the first measurements of the vector change of polarization at different locations around the shower axis. This latter property in particular is unique to radio Čerenkov detection, and the combination of all these results gives us confidence that the road to Teraton neutrino detectors is still wide open along this approach.

\section{EXPERIMENTAL SETUP}

The experiment (SLAC T460) was performed at the Final Focus Test Beam (FFTB) facility at SLAC in June 2002. As in our first SLAC measurement [17], we used gamma-rays from a series of Aluminum radiators of different thicknesses to provide secondary bremsstrahlung gamma-rays for shower production in our rock salt target. The geometry of the target is shown in Fig. 1. It was built of $1.8 \mathrm{~kg}$ salt bricks obtained from Morton Salt Inc., with a total mass of about 4 metric tons. The bricks are slightly trapezoidal in all of their cross sections to accommodate the manufacturer's mold release, but have average dimensions of $15 \times 10 \times 6 \mathrm{~cm}$. Such bricks are food-grade, and specified to be at least $99.5 \%$ pure sodium chloride. They are manufactured by compression molding of crystalline salt under $\sim 450$ tons per in ${ }^{2}$ pressure. Their density was measured to be $2.08 \mathrm{~g} \mathrm{~cm}^{-3}$, which is about $3 \%$ less than the density of pure halite mineral.

Measurements of the water content of small samples of a typical brick found only trace amounts of volatiles (including water), below $0.17 \%$. Laboratory measurements of sodium chloride crystals at comparable purities give upper limits of $\leq 10^{-4}$ on the loss tangent $\tan \delta$, which is related to the absorption coefficient $\alpha$, for small values of $\tan \delta$, by

$$
\alpha \simeq \frac{2 \pi \nu}{c} \sqrt{\varepsilon} \tan \delta\left(\mathrm{m}^{-1}\right)
$$

for frequency $v$, real part $\varepsilon$ of the dielectric permittivity, and speed of light $c$. The implied upper limits on the attenuation length $L_{\alpha}=\alpha^{-1}$ are hundreds of $\mathrm{m}$ [18] in the UHF and $\mathrm{cm}$ wave regime, and our tests through of order 0.3-0.4 m of the salt bricks found no measurable attenuation. In the results reported here we assume negligible bulk absorption of the radio emission. Paper labels attached to the the top of the bricks were found to be difficult to remove, and after confirming that they were non-conductive and had no effect on the radio transmission, they were left alone.

To minimize the effects of the slight gaps left when stacking the salt blocks, pure (food grade) un-iodized table salt was used to fill in the interstices. A $2.5 \mathrm{~cm}$ thick polyethylene sheet was also mounted along the Cherenkov radiator surface to provide a smooth refracting surface for the external antennas and some improvement in index matching because of the intermediate RF index of refraction of polyethylene.

An array of 7 by 3 (21 total) printed-circuit board (PCB) broadband dual-linear-polarization bowtie antennas was embedded in the upper portion of the target, on a rectangular grid with spacings of $22 \mathrm{~cm}$ along the shower axis and $21 \mathrm{~cm}$ transverse to it, with one set of antennas arranged with one of their polarization axes aligned directly above the beamline. These
PLAN VIEW (FROM ABOVE ANTENNA LAYER)

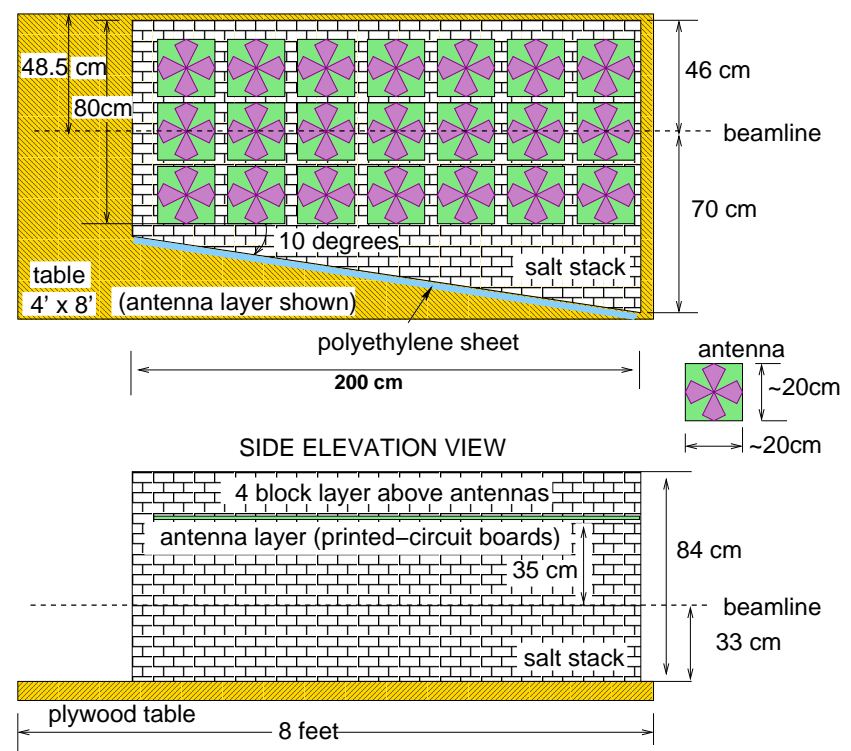

FIG. 1: Geometry of the salt-block shower target used in the experiment.

are shown schematically in Fig. 1. The antennas had a frequency response of approximately $0.2-2 \mathrm{GHz}$, and about -12 $\mathrm{dB}$ of cross-polarization rejection.

In addition to the embedded array, we also used two external antennas, a C/X-band standard gain horn usable over the frequency range from 5 to $9 \mathrm{GHz}$, at a fixed location on the side of the target; and a 1-18 GHz log-periodic dipole array (LPDA) antenna with a single linear polarization, which was also located to the side of the target but could be repositioned. One side of the salt stack was arranged with a $10^{\circ}$ angle with respect to the beamline to facilitate transmission of the Čerenkov radiation, which would have otherwise been totally internally reflected from a parallel face. The partially constructed salt stack is shown in Fig. 2 at the antenna layer, with several of the antennas evident, assembled with their leads projecting through a drilled salt brick.

All antenna signals were digitized with Tektronix digital oscilloscopes (a TDS694C $3 \mathrm{GHz}$ bandwidth, 10 Gsamples/s; and a CSA8000 sampling scope, $20 \mathrm{GHz}$, up to $50 \mathrm{Gsam}$ ples/s) using an ultra-stable microwave transition-radiationbased trigger from an upstream location. Runs were also taken with the bremsstrahlung radiators in and out of the beam at numerous times during the data collection to test for the presence of accelerator backgrounds, which were found to be negligible. The total shower energy was varied by changing both the electron beam intensity and the thickness of the bremsstrahlung radiators. These radiators varied from $0.06 \%$ to $1.5 \%$ of a radiation length and thus extracted the same fraction of the electron beam energy in bremsstrahlung photons. The electrons were then steered away from the salt target and dumped into the FFTB beam dump approximately $20 \mathrm{~m}$ downstream. The bremsstrahlung photon spectrum from the radiators used is very broad with a mean energy of several 


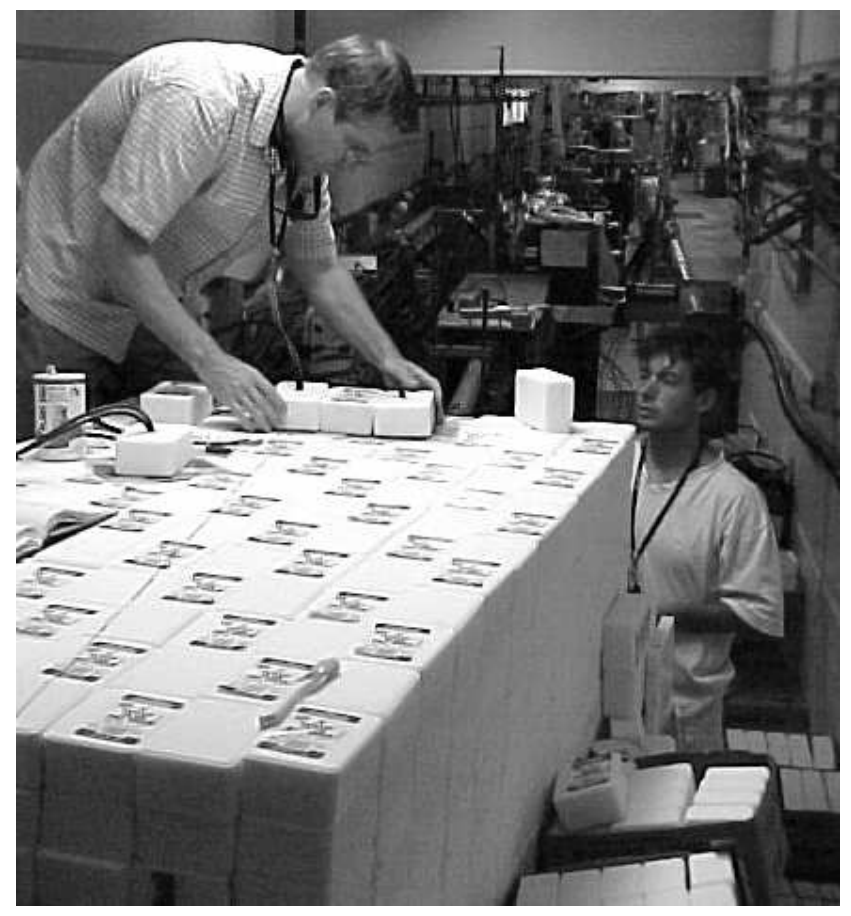

FIG. 2: View of the salt stack construction at the antenna layer. Antennas were mounted with their leads passing through drilled salt blocks as seen in several examples on the top of the stack.

$\mathrm{GeV}$.

SLAC beam monitors were able to reduce beam intensity to about $10^{9}$ electrons per bunch using conventional methods. Below this level, closed loop control (eg. fine-tuning) of the beam direction was not possible. However, by employing our high signal-to-noise coherent microwave transition radiation trigger, we were able to further monitor the beam intensity to levels an additional 2 orders of magnitude below this, and with the combination of radiators available to us, the dynamic range of the showers obtained was thus about 4 orders of magnitude in shower energy. The excellent stability of the FFTB beam assured that no retuning was necessary during our measurements, which were repeatable once the beam intensity was set back to the higher levels again.

The showers produced in such experiments consist of the superposition of a large number of $\mathrm{GeV}$ electromagnetic cascades whose energy sums up to EeV levels, but whose development is determined by the convolution of a $\mathrm{GeV}$ cascade and the exponential first-interaction distribution of the input photons. As such it cannot simulate the leading interactions of a cascade initiated by a single high energy particle, either in the longitudinal shower distributions of particle number, or in the exact particle species content. However, these differences are of little consequence for radio production, since the Askaryan emission is dominated by the electron excess at energies below $\sim 100 \mathrm{MeV}$, near the region of shower maximum. The charge excess itself is quite similar in either case, and the main quantitative differences come in only as the logarithm of the ratio of the lead particle energy to the critical

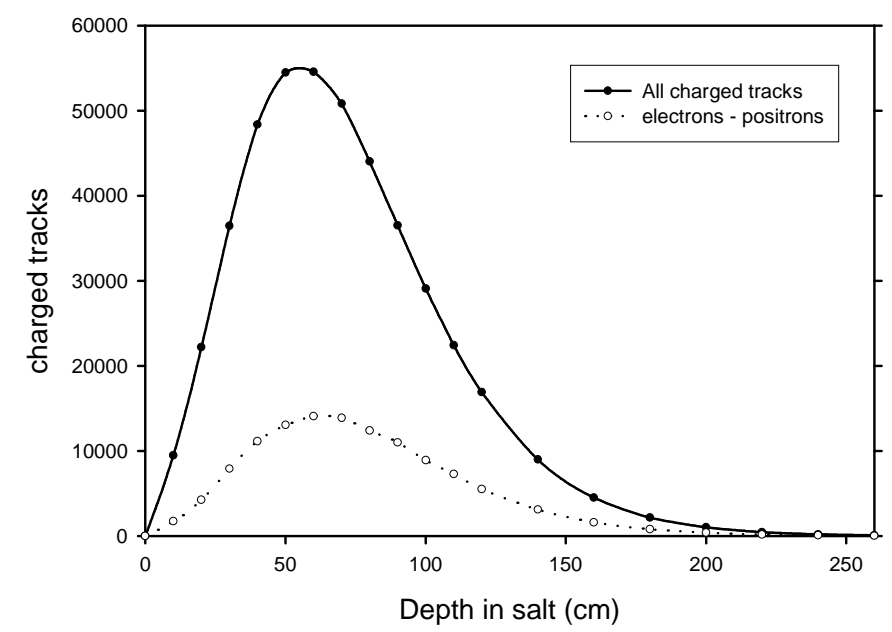

FIG. 3: Longitudinal distribution of the bremsstrahlung shower, for both total charge and charge excess as simulated by EGS4 for rock salt.

energy, which produces a more extended shower maximum region in the case of a shower initiated by a high energy particle, and gives more total track-length for Cherenkov production. These differences are easily quantified, and in practice the $\mathrm{GeV}$ composite showers used here can be used to rigorously test models for the Askaryan radio emission.

\section{RESULTS}

To provide a baseline for evaluation of the results, we simulated the electromagnetic showers using the Electron Gamma Shower 4 (EGS4) Monte Carlo code [19]. EGS4 cannot directly simulate the radio emission from the showers, but can assess both longitudinal and lateral development of the total charged particle and photon content of the shower, as well as the Askaryan charge excess.

Fig. 3 shows the longitudinal shower development for a salt target, including the charge excess. Fig. 4 shows a series of profiles of the shower lateral distribution of the excess charge at different depths in $\mathrm{cm}$, indicated on the plot. In each case the simulation is for $100028.5 \mathrm{GeV}$ electrons initially, led through a $10 \%$ bremsstrahlung radiator to fully account for the secondary photon spectrum. No changes are required to the EGS4 code to develop the $\sim 25 \%$ negative charge excess seen; this again demonstrates that the Askaryan effect is generic to electromagnetic showers.

The simulations of the shower transverse distribution were qualitatively confirmed by the appearance of a deposit of discolored material along a volume of salt within the stack during the experiment, the evidence of which is shown in Fig. 5. The size and shape of this cross section of the contaminated region is an indication of the size of the shower core itself, and it is still of order several $\mathrm{cm}$ in diameter even at $\sim 15$ radiation lengths deep. 


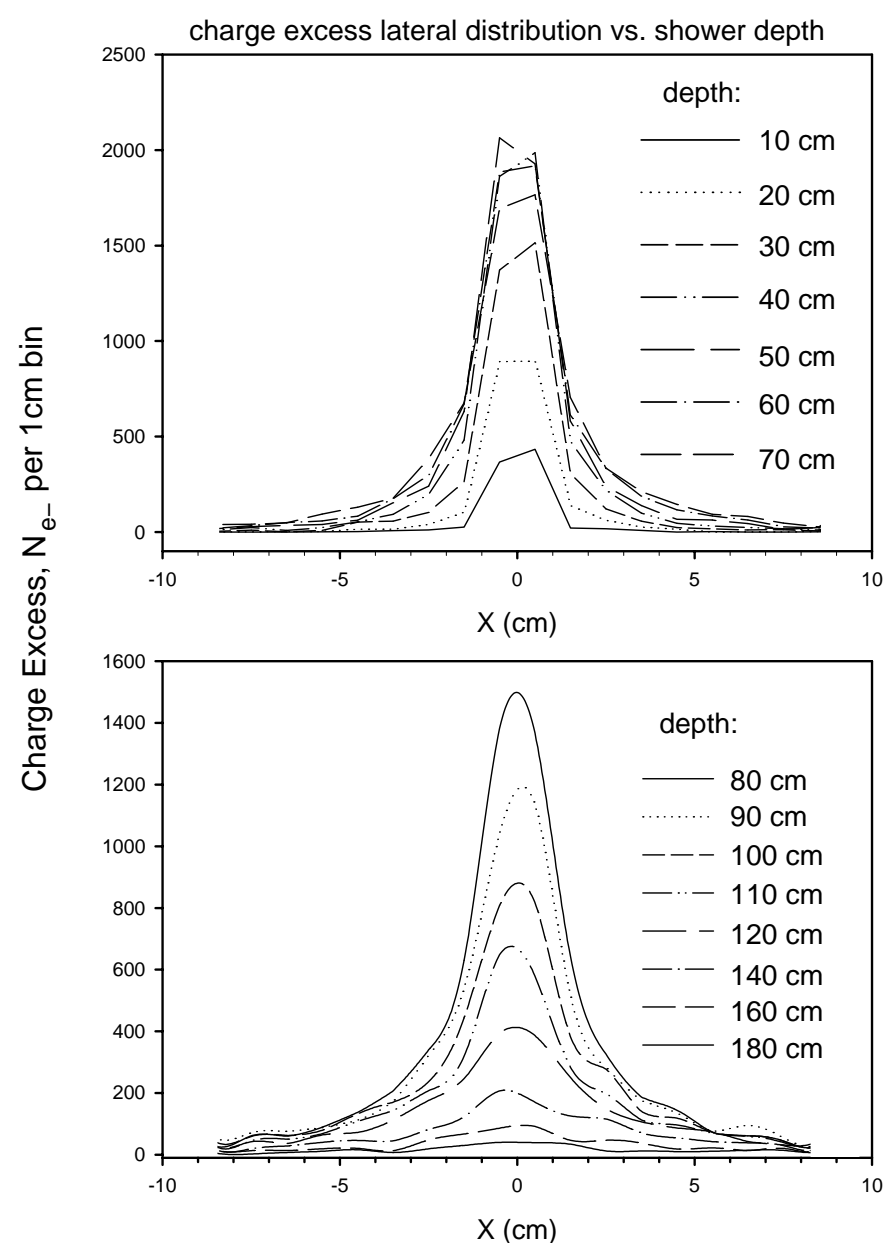

FIG. 4: Lateral distributions at various depths. Top: depths less than $70 \mathrm{~cm}$. Bottom: depths greater than $70 \mathrm{~cm}$.

\section{A. Coherence \& Absolute Field Strength.}

In reference [17], the coherence of the radiation was observed over a fairly limited range of shower energies, from about $3 \times 10^{17} \mathrm{eV}$ to $10^{19} \mathrm{eV}$ composite energy. In our current measurements, we have extended the coherence measurements to a much larger range. These measurements were made using averaged results from several of the bowtie antennas around the region of shower maximum. Typical runs at a given total charge per bunch (which determines the composite energy per bunch) were averages of 1000 beam shots, and the beam current was separately monitored for stability. Typically we also recorded 100-1000 shot-to-shot pulse profiles to monitor the variance. Once corrected for minor variations in beam current, these were stable to the level of a few percent.

Fig. 6 shows the results of the relative radio-frequency power as a function of shower energy, now covering nearly 4 orders of magnitude in energy and 8 orders of magnitude in RF power. No departures from the expected quadratic dependence of pulse power with shower energy are seen. This result demonstrates the remarkable dynamic range possible for de-

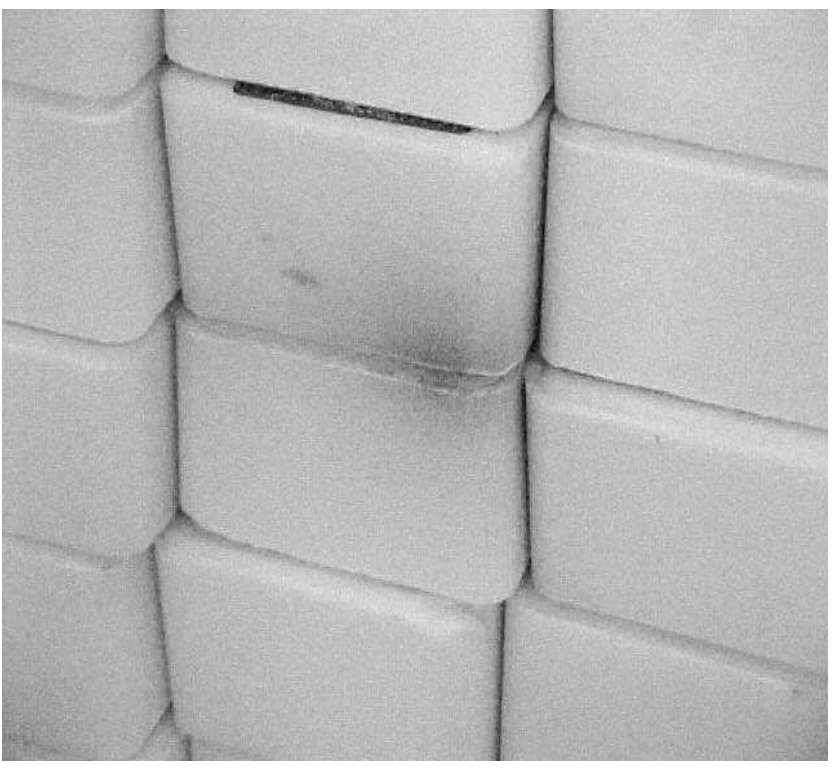

FIG. 5: The exit point of the beam in the downstream wall of the salt target, outlined by discoloration of the salt produced by beam and showing in cross section at the exit surface. Even at this location of order 15 radiation lengths along the shower, the shower core is still only several $\mathrm{cm}$ across (the salt blocks are 6 by $10 \mathrm{~cm}$ here in cross section).

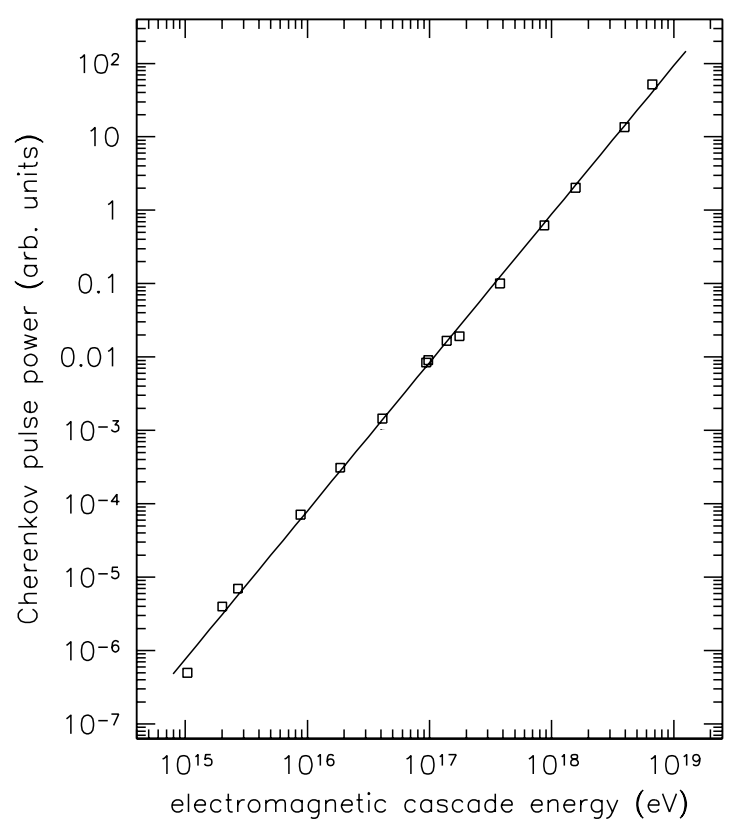

FIG. 6: Observed coherence of the $0.3-1.5 \mathrm{GHz}$ radiation as a function of total beam energy per pulse. The curve shows a quadratic relation for power as a function of shower energy. 

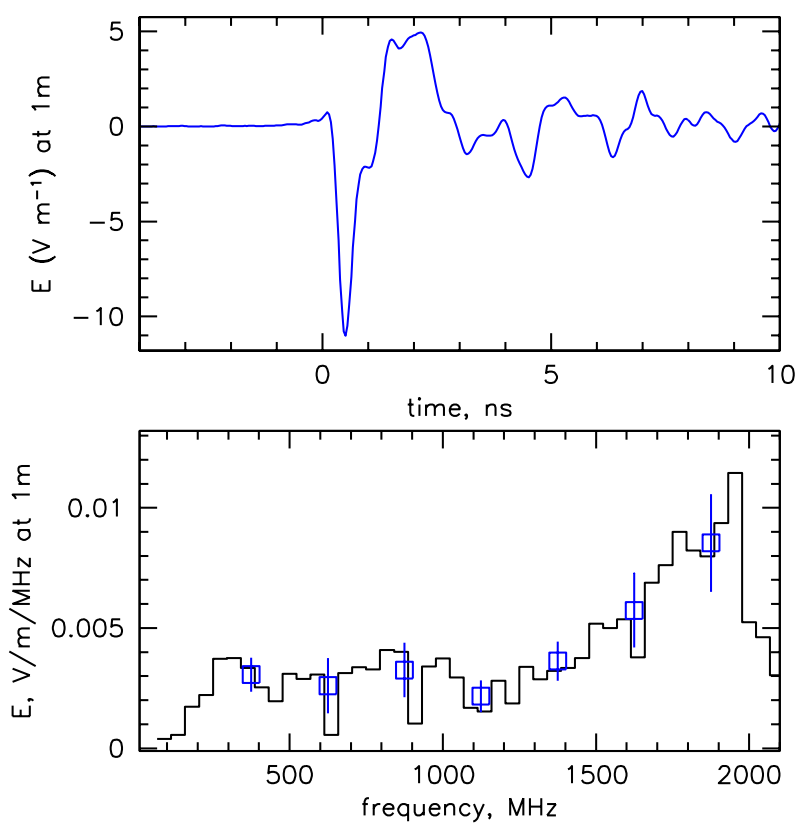

FIG. 7: Top: summed-in-phase pulse profile from all bowtie antennas. Bottom: absolute power spectrum of the bowtie data, corrected for antenna angular response.

tection of coherent RF Čerenkov radiation.

Determination of the absolute measured field strength of the showers requires that the antenna effective area, spectral response, coupling efficiency, and angular response all be included in converting the measured voltages to field strengths in the detection medium. To properly account for the angular response, one must first determine if the conditions for the reception are near-field (Fresnel zone) or far-field (Fraunhofer Zone) for both the antenna and radiation source. The conditions for far-field response are approximately

$$
f \leq \frac{R c}{L^{2} \cos ^{2} \theta}
$$

where $R$ is the distance between source and antenna, $L$ the largest antenna dimension, $\theta$ angle of incidence with respect to the normal (broadside) of the antenna, and $f$ is the highest frequency for which far-field conditions are met. For the antenna as a receiving component, with $R=38 \mathrm{~cm}, L=20 \mathrm{~cm}$, and $\theta=25^{\circ}$ at the Čerenkov angle the antenna far field conditions are satisfied for frequencies below $2 \mathrm{GHz}$, the highest usable frequency for the bowtie antennas.

While the shower is in the far-field of the antenna, the converse is not the case, since the radiation develops over a region comparable in size to the entire shower with a length of about $80 \mathrm{~cm}$ for full-width-at-half-maximum near the peak of cascade particle development. In practice this means that while the far-field antenna beam patterns may be used to determine the bowtie amplitude response with angle, they receive radiation only from approximately that portion of the shower that is projected onto the antenna effective area at the Čerenkov an- gle (about $66^{\circ}$ ). This behavior was observed in paper [17], and provides the possibility of tracing out the longitudinal charge evolution of the shower by measuring the change in radiation amplitude along the shower axis, as was done in paper [17] (see also Fig. 10 below). In determining the absolute field strength one must therefore account for the fact that the shower is effectively resolved into these individual sections.

Fig. 7(top) shows a plot of the summed field strengths from all of the bowtie antennas, corrected for known cable attenuation but not for other frequency dependent effects. The pulse shape is typical of the impulse response for bowtie antennas. In the bottom portion of the figure, a Fourier amplitude spectrum of the field strength is shown, including corrections for the gain of the bowtie antennas. The solid histogram shows the power spectrum of the pulse, and the points are averages over $250 \mathrm{MHz}$ sections of the spectrum, with the error bars based on the spectral variance within each section. It is evident that there are some near-nulls in the bowtie response, but the overall trend is for a rise at the higher frequencies, consistent with Čerenkov radiation.

We also measured the coherence at higher frequencies (2.2$15.0 \mathrm{GHz}$ ) in several bandpasses, using the LPDA and the C/X-band horn antennas, both of which had known effective areas. Fig. 8 shows the measured electric field strength vs. total shower energy at different frequencies. Measurements were done with the LPDA on 4.95 and $14.5 \mathrm{GHz}$ respectively and with the horn antenna at $7.4 \mathrm{GHz}$. Points represent measured values and lines are least-square fit curves $|\mathbf{E}|=A E_{s h}{ }^{\alpha}$, where $\mathbf{E}$ is the electric field and $E_{s h}$ the shower energy. The fit for the exponent $\alpha$ gives: $\alpha_{4.95}=1.00 \pm 0.04$ at $4.95 \mathrm{GHz}$, $\alpha_{14.5}=1.02 \pm 0.11$ at $14.5 \mathrm{GHz}, \alpha_{7.4}=0.99 \pm 0.05$ at 7.4 GHz. On all three frequencies, the field strength is in agreement with full coherence of the radiation.

Fig. 9 shows the absolute field strength measured in several frequency bands from 0.3-15.0 GHz. The plotted curve is based on the parametrization given in [20,21], scaling from ice to synthetic rock salt (for details see Appendix B). The horizontal bars are not standard errors but show antenna or filter bandwidth, and vertical bars indicate combined statistical and systematic uncertainties. The measured spectrum of the pulse electric field is in good agreement with the prediction.

\section{B. Polarization and charge excess tracking}

The crossed bowtie antennas employed in our measurements provided simultaneous measurements of the induced voltage in orthogonal linear polarizations, but because of their geometry, there is inherent leakage of a co-polarized signal into the cross-polarized receiver. With a measured $12 \mathrm{~dB}$ of cross-polarized rejection, up to $15 \%$ of the co-polarized amplitude (eg. voltage) can appear in the cross-polarized channel. To make accurate estimates of the angle of the projected plane of polarization of the radiation, the polarization leakage must be accounted for. We use a simple model for this, where the co- and cross-polarized voltages are related by:

$$
V_{0}=\mathbf{E} \cdot\left[\mathbf{h}_{\mathbf{0}}+\alpha \mathbf{h}_{\mathbf{9 0}}\right]
$$




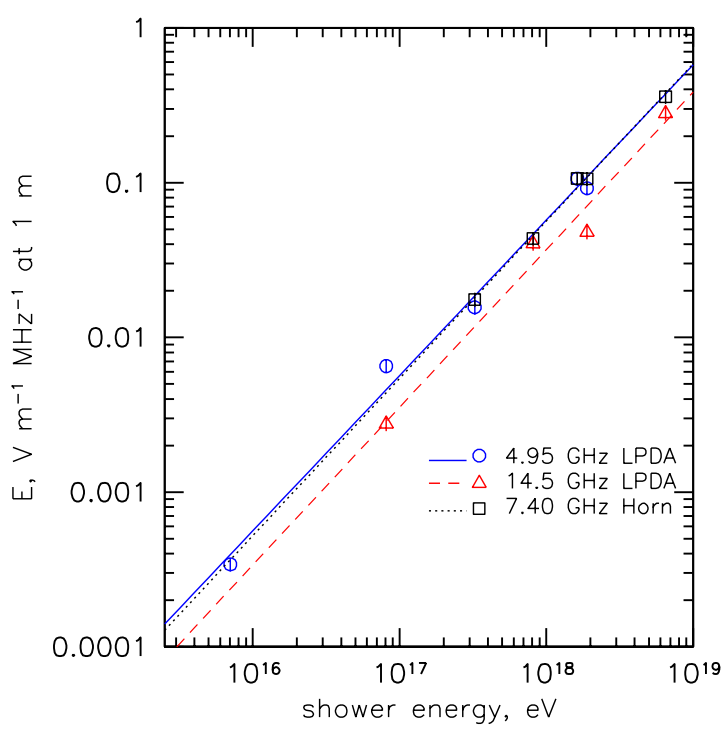

FIG. 8: Measured coherence of electric field strength at 4.95, 14.5 with LPDA and $7.4 \mathrm{GHz}$ with horn antennas respectively, with leastsquares fit curve.

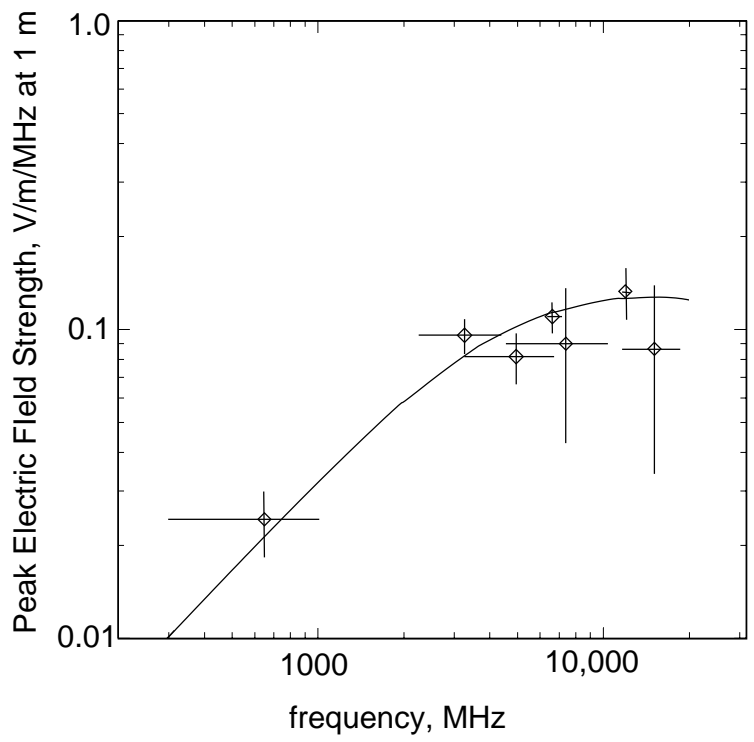

FIG. 9: Spectral dependence of the measured electric field strength for shower energy $1.9 \times 10^{18} \mathrm{eV}$. The solid curve is a parametrized Monte Carlo simulation scaled for synthetic rock salt. Vertical bars are estimated errors, largely due to systematics in the absolute RF calibration; horizontal bars indicate the bandwidth used.

$$
V_{90}=\mathbf{E} \cdot\left[\mathbf{h}_{\mathbf{9 0}}+\alpha \mathbf{h}_{\mathbf{0}}\right]
$$

where $\mathbf{E}$ is the electric field vector of the radiation and $\mathbf{h}_{\mathbf{0}, 90}$ are the vector effective heights (which are complex in general) of the co- and cross-polarized antennas. Since each of the bowtie antennas are identical in both the co- and crosspolarized directions, and since the induced voltage has a simple sinusoidal dependence on the projected plane of polarization angle $\Psi$ (assuming no net circular polarization), we can write

$$
\begin{aligned}
V_{0} & =E h(\cos \Psi+\alpha \sin \Psi) \\
V_{90} & =E h(\sin \Psi+\alpha \cos \Psi)
\end{aligned}
$$

where $h=\frac{1}{2}\left(\left|h_{0}\right|+\left|h_{90}\right|\right)$ has the same magnitude for both the co- and cross-polarized antennas.

In our case we choose the co-polarized direction to be aligned with the beam axis, since the radiation is naturally expected to be polarized along this axis. If we assume that $\Psi$ is approximately aligned with the beam axis (in practice this was done to a precision of about $1-2^{\circ}$, then for an antenna along the beam axis, we find to a good approximation that $\alpha=\left.\left(V_{90} / V_{0}\right)\right|_{\text {on-axis. }} \Psi$ is then determined by

$$
\Psi=-\tan ^{-1}\left(\frac{\alpha-V_{90} / V_{0}}{\alpha V_{90} / V_{0}-1}\right) .
$$

Fig. 10 shows results of the polarization and relative amplitude measurements for all 21 of the bowtie antennas. The top pane plots these as vectors with scaled lengths and directions corresponding to the square root of the amplitude (used for clarity for the lower amplitudes) and the projected plane of the polarization $\Psi$. In the lower pane of the figure, we also show the relative amplitude of the antennas along the center axis as a function of the longitudinal distance along the shower (corrected for the Čerenkov angle projection). We also plot the EGS4 simulation of the shape of the charge excess along the shower (about $27 \%$ of the total charge). There is excellent agreement between the measured shape of the amplitude response and the normalized charge excess predictions from EGS4.

In Fig. 11 we show similar data for the three antennas at shower maximum at a depth of $50 \mathrm{~cm}$, now plotting the plane of polarization as a function of the transverse position of the antenna with respect to the centerline of the beam. All of the antennas lay in a plane about $35 \mathrm{~cm}$ above the beamline. The solid curve shows the expected change in the angle of polarization for the three antennas, and the agreement is good.

\section{Transition radiation}

Transition radiation (TR) and Čerenkov radiation are closely related; this is particularly true in the radio regime, where both forms of radiation may coexist in partially or fully coherent forms, and interference between them may result. In earlier work [22] we demonstrated the existence of free-space coherent microwave TR from relativistic electron bunches exiting an accelerator beam pipe, and confirmed the predicted 


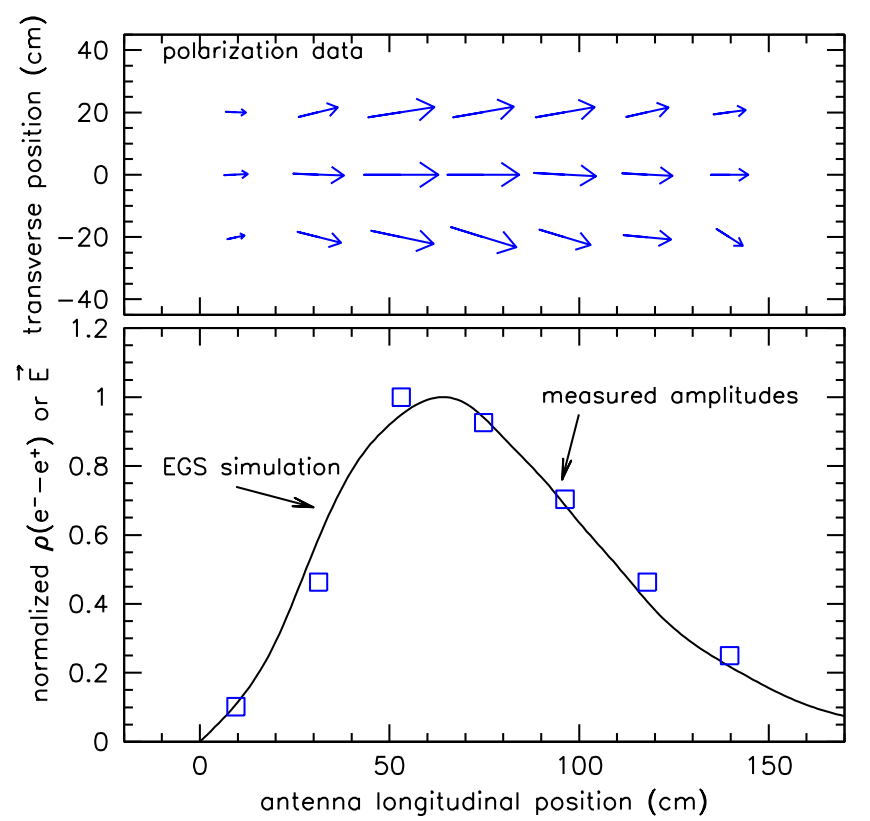

FIG. 10: Top: Measured polarization vector field at the bowtie antenna locations in a plane $34 \mathrm{~cm}$ above the shower axis. The antenna feed center locations in that plane give the base of each vector, which is scaled in length as the square root of amplitude for clarity. The angles of the vectors match the observed polarization angles. Bottom: The observed amplitude of the received RF pulses along the center line of the antenna array. Also plotted is a curve of an EGS simulation of these showers; both curves are normalized to unit peak amplitude.

angular dependence and polarization of the radiation. However, there was a serious discrepancy in these earlier measurements with regard to the total emitted power, which was observed to be significantly lower than predictions.

Transition radiation in the radio regime is of particular interest for detection of high energy particle showers, since the Askaryan charge excess guarantees not only coherent Čerenkov radiation, but also TR when there is any change in the dielectric medium on scales comparable to the size of the shower. Showers which break through a solid surface can produce forward TR in the direction of propagation, and showers originating in one medium (air for example) which then intersect a solid surface will produce backward TR which propagates opposite to the original shower direction. It is thus of some importance to establish the emitted power from this process, to determine under what circumstances it may be useful for high energy shower detection.

In a setup that was independent of the salt target, and done after the work with the salt was complete, we used the photon beam to produce an electron-gamma shower in a $2 \mathrm{~cm}$ block of lead followed by a $1.3 \mathrm{~cm}$ block of aluminum. The excess electrons from the shower are thus expected to produce TR as they exit the aluminum. Strong RF pulses were observed with an X-band horn, with typical duration of $0.45 \mathrm{~ns}$, con-

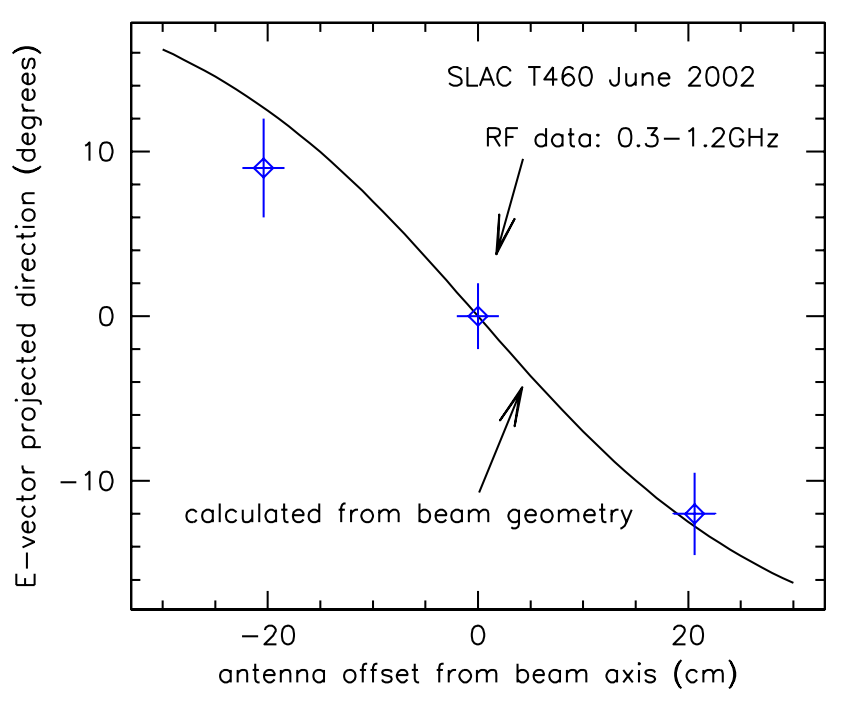

FIG. 11: Plane of polarization vs. transverse distance of the antennas, which were all about $35 \mathrm{~cm}$ above the axis of the beam.

sistent with a completely band-limited signal $\left(h_{e f f}=2.4 \mathrm{~cm}\right.$, $\langle\lambda\rangle=4.2 \mathrm{~cm}, d v=2 \mathrm{GHz}$, effective area $A_{\text {eff }}=18.87 \mathrm{~cm}^{2}$ ). An EGS simulation provided us with an estimate of the number of excess electrons, their energy, and their angular and transverse distributions, which are shown in Fig. 12. In an appendix, we provide details of the calculation of the expected TR power, which depends on the geometric form factors of the exiting bunch. The transverse and angular divergence parameters are estimated to be $\sigma_{T}=6 \mathrm{~mm}$ and $\Omega=30^{\circ}$, respectively; therefore $f_{T}=0.996$ and $\chi=0.08$. Most of the shower electrons are in the highest energy bin $(0.975<\beta<1)$, for simplicity we use only the electrons in that bin for this calculation, setting the average $\beta=.9875$. We assume the longitudinal form factor $f_{L}=1$ which is reasonable given the very tight longitudinal distribution of SLAC bunches.

The results of the TR run are shown in Table I.

TABLE I: TR measurements \& analysis.

\begin{tabular}{|c|c|c|c|c|c|c|c|}
\hline \hline$\theta$ & $\begin{array}{c}\mathrm{D} \\
\mathrm{cm}\end{array}$ & $\begin{array}{c}d \Omega \\
\mathrm{msr}\end{array}$ & $N_{e}$ & $\begin{array}{c}P_{L} \\
\mathrm{~mW} \\
\text { (calc.) }\end{array}$ & $\begin{array}{c}P_{L} \\
\mathrm{~mW} \\
\text { (meas.) }\end{array}$ & $\begin{array}{c}E \\
\mathrm{~V} / \mathrm{m} \\
\text { (calc.) }\end{array}$ & $\begin{array}{c}E \\
\mathrm{~V} / \mathrm{m} \\
\text { (meas.) }\end{array}$ \\
\hline $10^{\circ}$ & 135.5 & 1.03 & $5.3 \times 10^{8}$ & 0.26 & 0.50 & 0.28 & 0.31 \\
\hline \hline
\end{tabular}

Here, the predicted power is about half the measured power, much closer than in our previous result [22]. The predicted electric field is $90 \%$ of the measured electric field. We would expect it to be about $70 \%$ of the measured electric field based on the factor of 2 difference in the power, but the electric field 

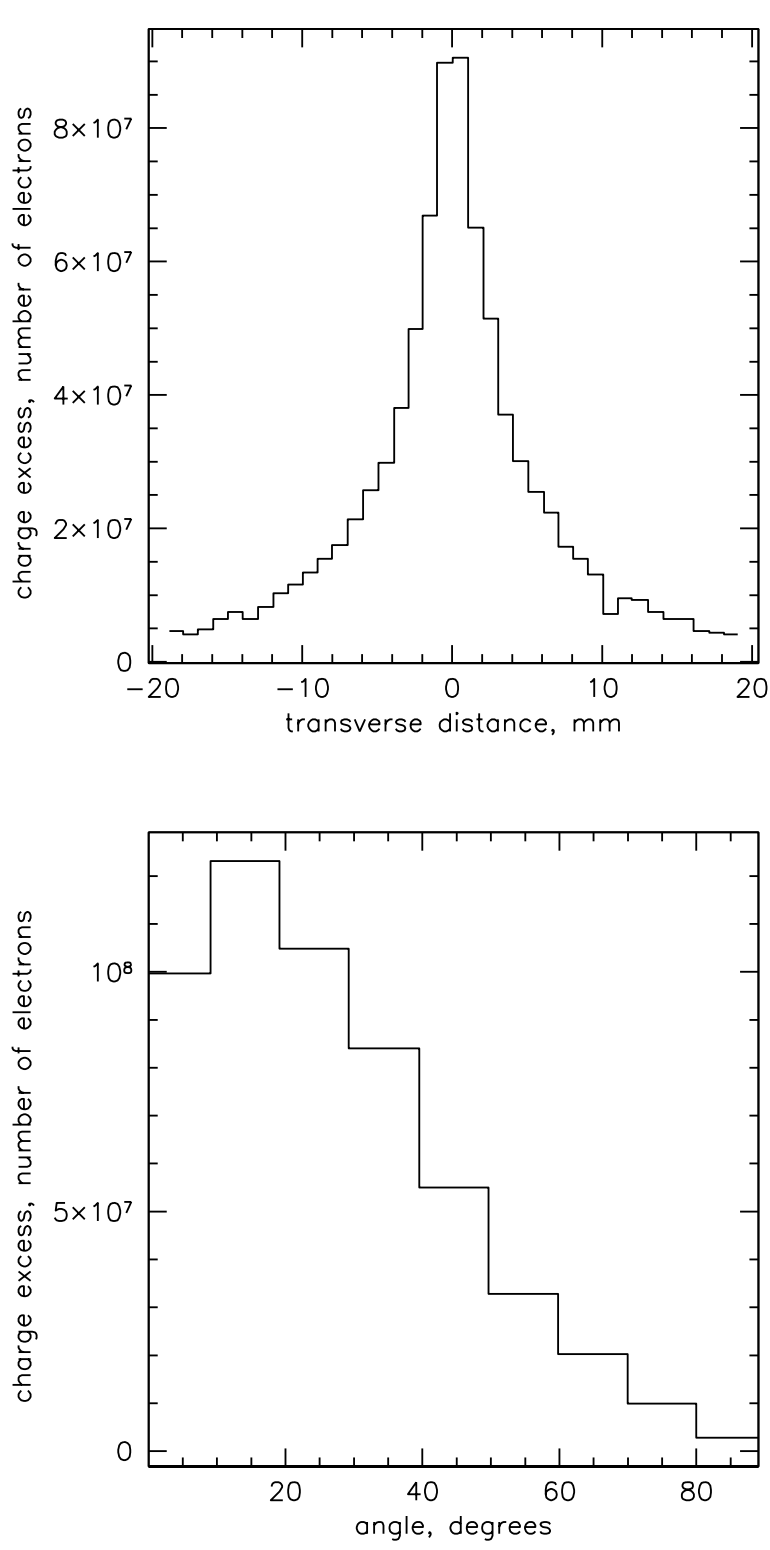

FIG. 12: Top: Transverse spread in the simulated electron shower emerging from the TR radiator, grouped in $1 \mathrm{~mm}$ annular bins around the beam axis. Bottom: angular emittance spread in the same electron shower, for the highest energy bin $(0.975<\beta<1)$, in annular bins of solid angle, with $10^{\circ}$ angular width.

estimate assumes a perfectly triangular envelope, which is not accurate. Detailed studies indicate that the peak and RMS voltages are related by $V_{R M S}=k V_{p} / 2 \sqrt{2}$, where $k \approx 1.4$ [23].

\section{DISCUSSION}

Our measurements have extended the validity of the theory underlying the Askaryan effect to a second dielectric material, with $30 \%$ higher density compared to silica sand, and a
$40 \%$ higher dielectric constant. In all cases the theory appears to scale as expected by the material properties, and there is no reason to believe this scaling would not apply for other dielectric materials as well. The measurements of transition radiation from the charge excess are the first observations of this effect, and lends further strength to the Askaryan hypothesis that a negative charge excess is responsible for the coherent radio emission, for if this were not the case, there is no obvious way to produce the measured transition radiation with the strength observed.

This latter effect could be important for both cosmic ray shower and neutrino shower detection, since there are many physical situations where a shower encounters a discontinuity of some kind that can lead to transition radiation. For example, ultra-high energy air showers encountering clouds will see a dielectric discontinuity; and giant air showers which impact the earth or the surface of the ocean will certainly produce strong backward transition radiation, particularly in the latter case where the Fresnel reflection coefficient from the surface of the ocean is of order unity.

Our radio Čerenkov measurements demonstrate conclusively that coherent Čerenkov radio pulses from showers in the rock salt can accurately reconstruct the profile of the shower development as well as the total shower energy. They show that the process is inherently quadratic in the rise of power vs. shower energy, and this relationship is reliable over many decades of these parameters. Combining these results with in situ measurements of propagation characteristics at radio frequencies in salt domes [16] which indicate $\geq 250 \mathrm{~m}$ attenuation length, we conclude that nothing in principle prevents the development of embedded antenna arrays within large salt structures such as salt domes for the characterization of ultra-high energy neutrino fluxes.

\section{A. Application to detector modeling.}

To further understand how this technique might be applied, we have created a Monte-Carlo simulation of a large-scale antenna array embedded within a generic salt dome, where the halite with the highest purity is likely to reside. We denote the array here as a Salt dome Shower Array (SalSA). The array is cubic with 1728 antenna nodes $(12 \times 12 \times 12)$, with a grid spacing of $225 \mathrm{~m}$, of the same order as conservative in situ estimates [16] of the field attenuation length in rock salt, which we assume to be $250 \mathrm{~m}$ in this simulation. In fact recent laboratory measurements using a dielectric cavity [15] on samples of rock salt from Hockly salt dome favor $L_{\alpha} \simeq 900 \mathrm{~m}$ at $200 \mathrm{MHz}$, and similar long attenuation lengths are also favored by ground-penetrating radar data [16].

Each node consists of 12 closely spaced antennas $(0.75 \mathrm{~m}$ vertical offset), 6 of which are dipoles with vertical polarization, and 6 of which are slotted-cylinder antennas which are sensitive to horizontal polarization. The antennas are both assumed to have $\cos ^{2} \theta$ dipolar response functions for simplicity here. The assumption is accurate for fat dipoles or bicones in the vertical polarization [26]; for our slot cylinders, the response in practice is somewhat more flattened than $\cos ^{2} \theta$ 
with a 1-2 dB front-to-back asymmetry, but we anticipate further development of current designs will tend to converge on a more uniform dipolar response.

Multi-antenna local nodes such as this are effective in enabling a lower local-trigger threshold in the presence of Gaussian thermal noise, and are very cost effective since the antenna elements themselves are inexpensive. The antennas are based on Numerical Electromagnetics (NEC2) models and actual tested prototypes, and are optimized for frequencies centered at about $200 \mathrm{MHz}$, with bandwidth of about $100 \%$. Beam patterns for the mid-frequencies are assumed.

A system temperature of $450 \mathrm{~K}$ is assumed, based on about $310 \mathrm{~K}$ for the salt and a receiver noise temperature of $140 \mathrm{~K}$, consistent with low-noise amplifiers that are readily available commercially. The basic design for node electronics centers on the use of a switched-capacitor array (SCA) transient digitizer, which is only read out and sent to the surface when the local node is triggered; a prototype of the basic design, which uses Gigabit ethernet on one fiber per node to the surface, is described by G. Varner et al. [24, 25].

\section{Hardware trigger rates.}

For triggering, an event must produce voltages above $2.8 \sigma$ on 5 of 12 of the local antennas within a node. This threshold and coincidence level is chosen both to reduce the accidental rates and to ensure a robust signal for reconstruction of the event. The accidental rates are addressed in the following discussion, and although we have not yet simulated the reconstruction process in detail, the five $2.8 \sigma$ signals yield a joint probability corresponding to $5.7 \sigma$, and even assuming one of the five is excluded for any reason, the remaining four signals have a joint $5 \sigma$ probability.

The propagation time across the $9 \mathrm{~m}$ high node requires that the time window for such a coincidence be about $80 \mathrm{~ns}$, corresponding to about 8 band-limited temporal modes of the antenna input noise voltages $(\tau=2 / \Delta f)$. For these conditions, random coincidence rates per node per coincidence window are given by the cumulative binomial probability density function:

$$
P\left(p, k, N_{\tau}\right)=\sum_{k}^{N_{\tau}} \frac{N_{\tau} !}{k !\left(N_{\tau}-k\right) !} p^{k}(1-p)^{N_{\tau}-k}
$$

where $p$ the individual antenna probability of exceeding a given voltage threshold in the window, $k$ the number of antennas required above threshold, and $N_{\tau}=\frac{T}{\tau} N_{\text {ant }}$ is the product of the number of antennas $N_{a n t}$ and the number of independent temporal modes of length $\tau$ within a coincidence window. Since antenna noise voltages obey a nearly Gaussian voltage distribution [26]

$$
p=\frac{2}{\sqrt{2 \pi}} \int_{2.8}^{\infty} e^{-x^{2} / 2} d x=0.0255 .
$$

For bandwidths of $\Delta f \simeq 200 \mathrm{MHz}, \tau=2 / \Delta f \simeq 10 \mathrm{ns, \text {and }}$ thus the number of possible "cells" that can combine to form a node trigger is $N_{\tau}=8 \times 12=96$. The resulting probability

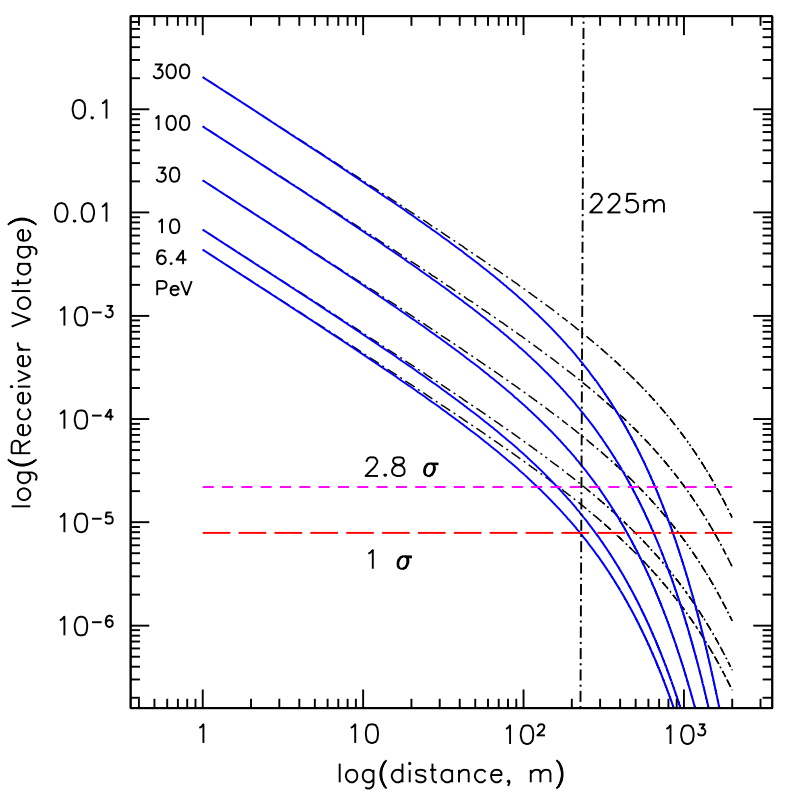

FIG. 13: Detection threshold estimates for a Saltdome Shower Array (SalSA). Solid \& dashdot curves show received voltages as a function of distance for a $250 \mathrm{~m}$ (solid) and $900 \mathrm{~m}$ (dashdot) attenuation lengths, for the energies marked at left. The 1,2.8 $\sigma$ noise levels shown are for a $450 \mathrm{~K}$ system temperature. The $225 \mathrm{~m}$ line is the antenna node spacing value used for the Monte Carlo.

of a single node trigger per $80 \mathrm{~ns}$ is $P_{\text {node }}=0.013$, yielding a node random trigger rate of about $R_{\text {node }}=130 \mathrm{kHz}$.

Since this rate is too high for sustained transient digitizer throughput, an additional digital signal processor is used to reduce the rate by first-order causality constraints. To enforce causality for the local antenna node triggers, we require that the hits along a node evolve according to a proper light cone. Considering a loose constraint using four $20 \mathrm{~ns}$ subintervals of the 80 ns coincidence window, the hit pattern may be thought of as consisting of a (12 antenna $\times 4$ subinterval) matrix of antenna hits vs. time.

The local trigger accepts any pattern of 5 or more hits within these 48 cells. The vast majority of random triggers will have no more than 5 hits, and there are

$$
\left(\begin{array}{c}
48 \\
5
\end{array}\right)=\frac{48 !}{5 !(48-5) !}=1.95 \times 10^{5}
$$

possible 5-hit patterns. Of these patterns, only those having a linear relationship of delay vs. antenna location are causal, giving of order 32 different combinations. Requiring causality thus implies a net rate of

$$
R_{\text {node }}^{C} \simeq R_{\text {node }} \times \frac{32}{1.95 \times 10^{5}} \simeq 20 \mathrm{~Hz}
$$

which provides ample margin on the expected $\sim 1 \mathrm{kHz}$ throughput of the transient digitizer.

Once these local node events are transmitted to the surface, global triggers based on array-processing of event clusters are 
determined. An example of how this may be implemented is as follows. For each array node that produces a trigger, we require a minimum of 4 additional local node triggers among all of its nearest neighbors including all diagonals. For each of the 8 array corner nodes there are 7 neighbors, for each of the 120 edge nodes there are 11 , for each of the 600 face nodes there are 17, and for the remaining 1000 interior nodes there are 26. The light-crossing time in salt along the diagonal of an interior sub-cube of 27 nodes (consisting of a center node and its 26 neighbors) is $3.23 \mu \mathrm{s}$, or about 40 of the node trigger windows $T_{\text {node }}=80 \mathrm{~ns}$. For a simple random coincidence, there are $(27$ nodes $\times 40$ trigger windows $)=1080$ cells in the trigger matrix. In this case, we use equation 7 with

$$
p=P_{\text {node }}=T_{\text {node }} R_{\text {node }}^{C}=80 \mathrm{~ns} \times 20 \mathrm{~Hz}=1.6 \times 10^{-6}
$$

along with $\mathrm{k}=5$, and $\mathrm{N}=1080$.

The resulting cluster trigger probability for the $3.23 \mu$ s window is $P_{\text {cluster }}=1.27 \times 10^{-16}$, giving a net cluster trigger rate of $R_{\text {cluster }}=3.9 \times 10^{-11} \mathrm{~Hz}$. For the same 5-node criterion the corresponding probabilities and rates for the faces, edges, and corners are far lower. Thus the potential contamination of true events by random coincidences per year for the entire array is bounded by

$$
R_{\text {array }}=P_{\text {cluster }} \times N_{\text {interior }} \times 365 \times 86400=1.23 \mathrm{~Hz} .
$$

indicating that, even prior to global event fitting constraints, the contamination from randoms can be made negligible. In practice, either local thresholds or cluster trigger criteria can be relaxed in hardware to ensure a manageable random "heartbeat" rate of order several $\mathrm{Hz}$, and further constraints on cluster causality can be enforced in software. This will be done to ensure adequate real-time monitor of array instrument health.

\section{Thermal noise backgrounds.}

From above, causality requirements alone are adequate to eliminate random triggers from the neutrino candidate event sample. The additional information from polarization, amplitude gradient, and antenna waveform phase at each node will only further establish this conclusion. The strength of these constraints arises primarily from the fact that each node is not just a point detector, but provides significant directional information on its own. The important conclusion here is that the sensitivity of our implementation is limited only by our conservative event reconstruction requirements (which determine the 5 antenna +5 node coincidence levels), rather than random thermal noise backgrounds.

We stress that thermal noise backgrounds in radio Čerenkov detectors, while in some ways analogous to the through-going cosmic-ray muon backgrounds seen in lower-energy optical Čerenkov detectors such as AMANDA, are in fact quite distinct in practice, since they do not represent an irreducible background to the physics events, and cannot reproduce the characteristics of neutrino events. Atmospheric muon backgrounds in lower energy detectors are irreducible over some range of solid angle near or above the horizon. A more relevant comparison with thermal noise backgrounds can be made with downgoing muon events mis-reconstructed as upcoming neutrino-induced events in low-energy detectors. In this case, the frequency of mis-reconstructed events for the lower energy neutrino telescopes can be made negligible by choosing higher software thresholds, and the same is certainly true for radio detectors as well with regard to thermal noise backgrounds.

\section{Simulation results.}

Fig. 13 shows the dependence of the received voltage, based on the parametrization of reference [20,21], scaled for salt, as a function of distance and shower energy. The curves are labeled by shower energy in $\mathrm{PeV}$, and both the $1 \sigma$ and $2.8 \sigma \mathrm{lev}-$ els for rms voltage above the baseline noise level are shown. The solid and dash-dot curves are for $L_{\alpha}=250,900 \mathrm{~m}$ respectively.

It is evident that the $225 \mathrm{~m}$ spacing we have employed in our Monte Carlo here will give a shower energy threshold in the neighborhood of $30 \mathrm{PeV}$ for our baseline assumption of $L_{\alpha}=250 \mathrm{~m}$, and in practice we find that other loss factors and the neutrino interaction Bjorken y-factor (eg., inelasticity) contribute to push the optimal sensitivity to neutrino energies of order $100 \mathrm{PeV}$. Fig. 13 indicates that, although it may be possible to operate a SalSA at a low enough energy threshold to detect events at the 6.4 PeV Glashow Resonance for $W$-vector boson production via $v_{e}+e^{-} \rightarrow W^{-}$, the node spacing required is below $100 \mathrm{~m}$, implying an order of magnitude more array elements. Since, for a broad-spectrum neutrino source, the integral over the resonance is relatively small in terms of the number of events [27] compared to the continuum, the justification for the increased array density is not clear. In addition, the GZK neutrino spectrum peaks above $\sim 100 \mathrm{PeV}$. However, if $L_{\alpha} \gg 250 \mathrm{~m}$ as indicated by recent results, the energy threshold will be considerably lower, of order $10 \mathrm{PeV}$ at a spacing of $225 \mathrm{~m}$, and only a modest increase in array density required to achieve good sensitivity at the Glashow resonance.

The volume enclosed by the simulated array is approximately $15.6 \mathrm{~km}^{3}$ of salt, or about $34 \mathrm{~km}^{3}$ water equivalent mass. An additional $11.4(63) \mathrm{km}^{3}\left(24.9(137) \mathrm{km}^{3}\right.$ w.e.) is contained in the boundary of the array within one attenuation length (for $L_{\alpha}=250(900) \mathrm{m}$ ) of the outer wall, where we have indicated the perimeter values for the longer attenuation length in parentheses. These perimeter events will in general also yield events which are well measured when they illuminate a face of the array. The effective fiducial volume thus approaches $60(170) \mathrm{km}^{3}$ w.e. for an array that easily fits into the upper $\sim 3 \mathrm{~km}$ of a typical salt dome. The solid angle acceptance of the array includes the entire upper $2 \pi$ hemisphere, and extends about $10-15^{\circ}$ below the horizon, depending on neutrino energy. This implies of order $400(1190) \mathrm{km}^{3} \mathrm{sr}$ w.e. acceptance, with a threshold of order $10^{17} \mathrm{eV}$ or less. Clearly an accurate measure of the attenuation lengths is still an important factor in the design, but our goal here is to show that even conservative values for $L_{\alpha}$ give compelling results.

The simulation itself is a straightforward Monte Carlo in- 


\section{Shower energy $=10^{18} \mathrm{eV}$ neutrino direction: $a l t=43^{\circ}, a z=216^{\circ}$}
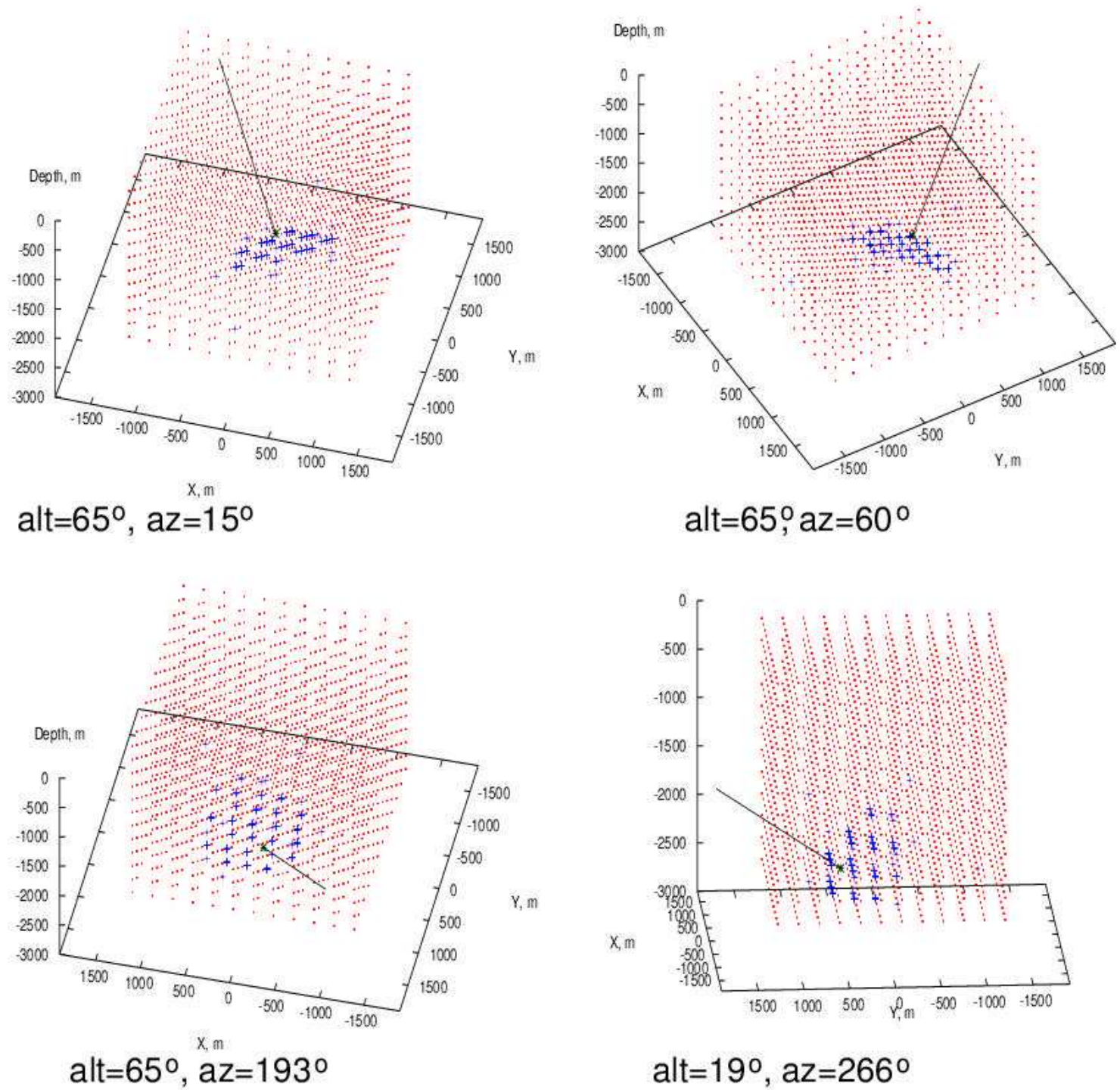

FIG. 14: Four views of the same $10^{18} \mathrm{eV}$ hadronic shower, with directions in local altitude and azimuth (counter-clockwise from east) shown. The dots mark antenna nodes, and crosses mark triggered nodes, with the incoming neutrino track shown up to its termination at the shower vertex.

tegral of the acceptance, using an isotropic neutrino flux, and integrating through a shell-density model for the earth to determine the angular distribution of neutrinos that can interact within the detector. We use a 1:1:1 $e: \mu: \tau$ mix of neutrino flavors, and include neutral and charged current events. For the radio emission, the Zas-Alvarez-Muñiz parametrization [20] is used. Full vector polarization is implemented. An noted above, we have not yet studied the event reconstruction precision, but this is not essential to determining the sensitivity, once the appropriate instrumental threshold is established.

In Figures 14, 15 we show 4 views each of two different events with shower energies of $10^{18}$ and $10^{19} \mathrm{eV}$. Here the dots locate antenna nodes, and those that produce triggers are marked with an 'x.' The incoming neutrino track is also indicated, with the shower occurring at its termination within the detector. The high refractive index of salt leads to a large Čerenkov angle of about $66^{\circ}$ and accounts for the apparent "flatness" of the cone of triggered regions around the event vertices. These events are not selected to be typical but rather to illustrate the detector geometry. Events of lower energy, while able to be reconstructed via both event geometry and polarization information, are not as easily discerned by eye. 


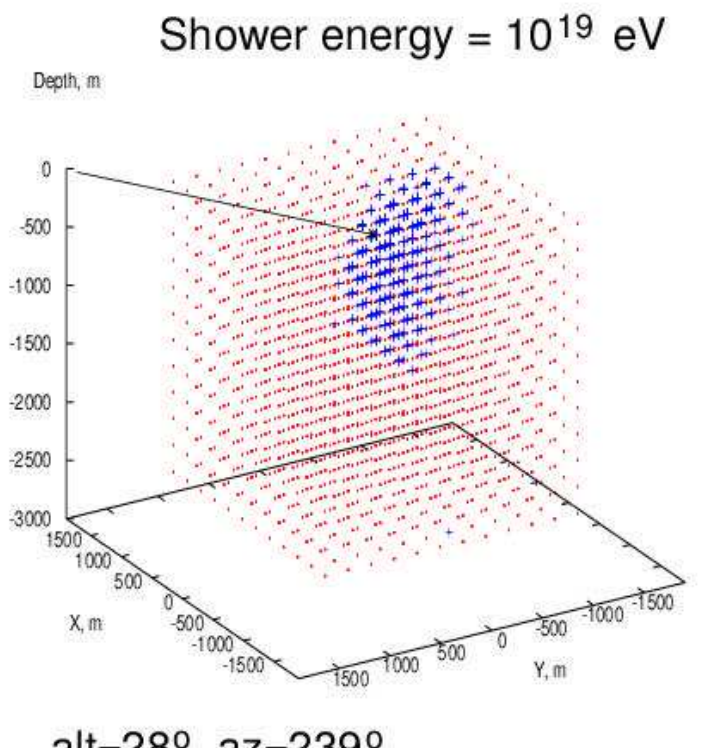

neutrino direction: alt $=8^{\circ}, a z=134^{\circ}$
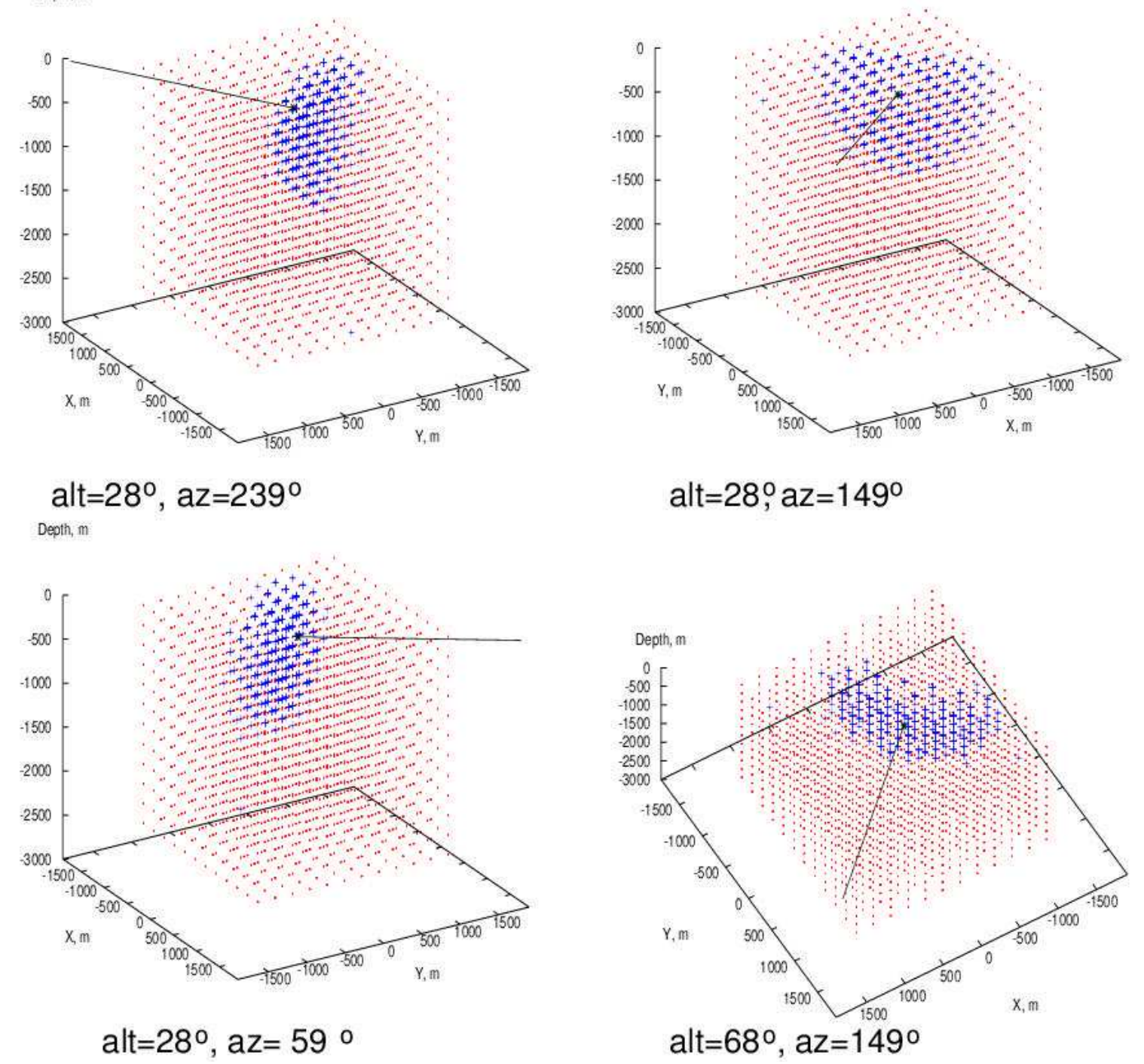

FIG. 15: Similar to previous, with shower energy $E_{s h}=10^{19} \mathrm{eV}$.

\section{Estimates of GZK neutrino event rates.}

The Monte Carlo is evaluated at discrete energies over the $\mathrm{PeV}$ to $\mathrm{ZeV}$ energy range, assuming a monoenergetic neutrino flux at each energy. The values of this function at discrete energies trace a smooth curve which represents a modelindependent estimate of the sensitivity for fluxes which are smooth compared to the energy resolution of the instrument, which is of order $\Delta E / E \simeq 1$, limited primarily by the uncertainty in inelasticity, which has a mean of order 0.23 at these energies and a standard deviation about equal to its mean. The resulting curve gives the acceptance, in $\mathrm{km}^{2} \mathrm{sr}$, which can be multiplied by the livetime to determine the exposure in $\mathrm{km}^{2} \mathrm{sr}$ s. The inverse of the exposure gives the model- independent flux sensitivity using the procedure outlined in ref. [28].

The result of this estimate for the flux sensitivity for 1 year of exposure is shown in Fig. 16, plotted along with various estimates of GZK neutrino fluxes [4, 29-31] for a complete range of possible parameters. Also plotted is an earlier SalSA sensitivity estimate [32] with a simpler detector model and more conservative trigger but otherwise similar methodology. For GZK neutrino models we have included estimates from four independent calculations, and in several of these cases we provide both minimal and maximal estimates where they were made.

For the three minimal GZK models shown the expected rates are about 10-12 events per year. If the observed rates are 


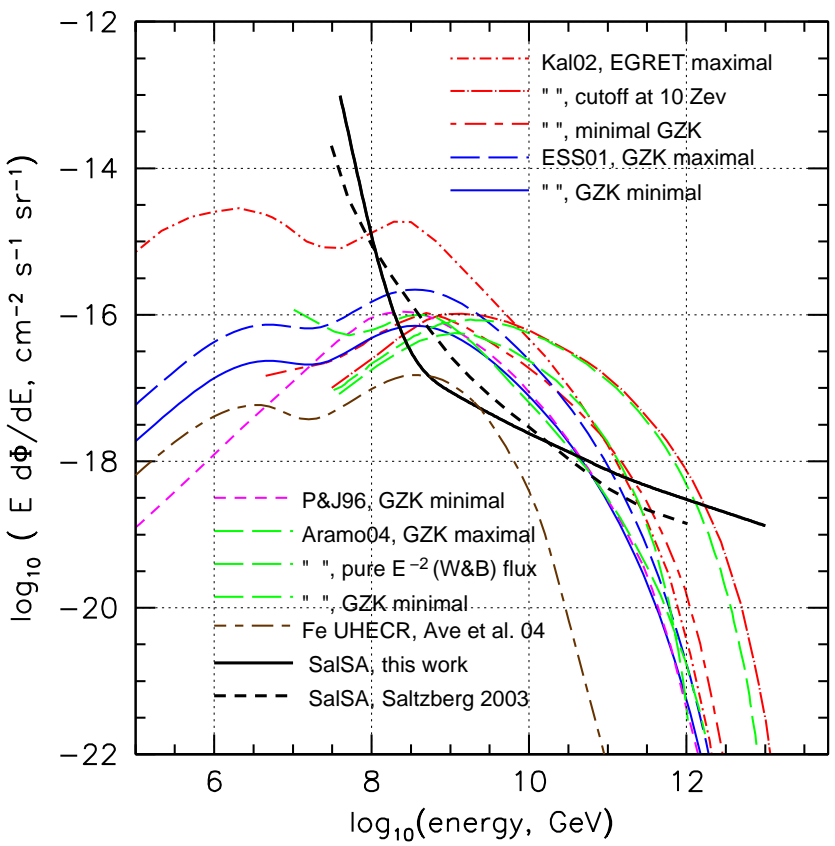

FIG. 16: Broad-spectrum flux sensitivity for a SalSA for one year of exposure, estimated from a Monte Carlo simulation. A complete set of GZK models is shown, as well as an earlier estimate of SalSA sensitivity [32]. From the highest to lowest the legend indicates fluxes from Kalashev et al. (2002) [29] for the maximal level allowed by EGRET limits, an intermediate model with a $10 \mathrm{ZeV}$ cutoff energy for the UHECR spectrum, and a minimal GZK model. The two models marked "ESS01" are from Engel et al. 2001 [4] for one minimal model and one with strong source evolution. The chosen P\&J96 model [30] is for a minimal GZK flux. Three different models are shown from Aramo et al. 2004 [31], a maximal model subject only to observational constraints, an intermediate model which follows a pure $E^{-2}$ spectrum as described by ref. [34], and another minimal GZK model. Finally, a model based on the unlikely assumption that the UHECR composition is pure iron is shown [33].

found to be significantly below this, the physics implications for both GZK neutrinos and cosmic rays would be serious as there are no currently acceptable models which predict rates below this. One class of models in which the UHECR are predominantly heavy nuclei such as iron have been considered [33], and such models produce a somewhat lower GZK neutrino rate, leading to between 3 to 10 events per year in our simulated detector. The assumption of no light nuclei among the UHECR is however purely ad hoc at this stage, and the data do not support such an inference, but the importance of this study is that it demonstrates that there are no currently known ways to evade the production of GZK neutrinos.

GZK neutrino models in the mid-range that assume stronger source evolution or a higher-energy cutoff to the cosmic ray source spectrum yield event SalSA rates in the range of 18-60 events per year; all of these models are still consistent with the Waxman-Bahcall limit for optically thin sources distributed cosmologically in a manner similar to AGN [34]. For the highest GZK neutrino models in which all parameters are maximized subject only to firm observational constraints such as the EGRET gamma-ray limits, the possible event rates are up to 100 per year.

We note that the event rates for the minimal GZK neutrino flux models, while still in the regime of small statistics, are likely to be measured with no physics background that we know of. The only possible background that we know of comes from charm quark production in ultra-high energy cosmic rays, which can decay to energetic secondary muons, which can then shower via either bremsstrahlung or photonuclear processes in the detector. However, even the highest current estimates [35] for this process yield throughgoing muon rates of $\leq 0.02$ per year above $10^{17} \mathrm{eV}$ over a $2.4 \mathrm{~km}$ radius area circumscribed around the detector volume. With regard to electromagnetic interference, even just several meters of overburden of rock or soil above a saltdome is enough to attenuate all anthropogenic radio or electronic interference, so that the system sensitivity will be subject only to the absolute thermal noise floor.

For a system with a design lifetime of 10 years or more, even minimal GZK neutrino fluxes will produce a rich data sample of events which would allow good precision on estimates of the GZK neutrino energy spectrum and sky distribution. In addition, the possibility of measuring secondary showers from the energetic leptons in charged-current events compared to neutral current events would allow for full calorimetry of the neutral and charged-current channels, since at $\mathrm{EeV}$ energies, secondary lepton are very likely to shower at least $10 \%$ of their energy within 1-2 km. LandauPomeranchuk-Migdal effects on the development and radio emission pattern from electron-neutrino events [36] can potentially allow flavor identification, as can decays of tau leptons produced in tau neutrino events [37].

Dramatic increases in neutrino cross sections above the standard model, such as might be produced by the effects of microscopic black hole production in theories with large extra dimensions [38], would yield much larger event rates than described here; several thousand events per year are then possible even for minimal GZK models. Events so produced would also have quite distinct character compared to the dominant charge-current deep-inelastic neutrino events, since the decay of the black hole by Hawking radiation leads to a pure hadronic shower with no secondary high energy leptons to produce more extended showers. There would also be no significant LPM effects in electron neutrinos, assuming the black hole production cross section dominated over the standard model cross sections. In fact, high cross sections combined with standard model GZK neutrino fluxes would serve to yield a detection with a proportionally smaller detector.

\section{CONCLUSIONS}

We conclude the following from our measurements and simulations:

1. The Askaryan effect behaves as theoretically expected in measurements made in synthetic rock salt. Corre- 
lation of the strength of the radiation to the estimated charge excess is excellent.

2. The polarization properties of the observed radio emission are consistent with coherent Čerenkov radiation, and can be used to derive geometric properties of the shower track to good precision.

3. The observed radiation is completely coherent over many decades of shower energy, and a wide range of radio and microwave frequencies.

4. Closely related transition radiation from the Askaryan charge excess of showers exiting dielectric or metallic media has been measured and found to be in agreement with predictions.

5. An antenna array of order $2.5 \mathrm{~km}$ on a side in an underground salt dome is well able to conclusively detect and characterize all current standard model GZK neutrino fluxes on even a 1 year time scale; over 10 years of operation, good statistics can be obtained on the GZK neutrino spectrum above $10^{17} \mathrm{eV}$.

\section{Acknowledgements}

We thank the staff of the Experimental Facilities Division at SLAC for their excellence and professionalism in support of our efforts, and Marc Rosen and his team of student technicians at the Univ. of Hawaii. This work was performed in part at the Stanford Linear Accelerator Center, under contract with the US Dept. of Energy, and at UCLA under DOE contract DE-FG03-91ER40662, and under DOE contract DE-FG0394ER40833 at the University of Hawaii. Both P. Gorham and D. Saltzberg are grateful for the support of DOE Outstanding Junior Investigator awards for studies in radio detection of high energy particles.

\section{Appendix A: Details of transition radiation analysis}

The forward spectrum of transition radiation is given in [22] as

$$
\frac{d^{2} W_{T R}}{d \omega d \Omega}=\frac{h \alpha}{2 \pi^{3}} \frac{\sqrt{\varepsilon_{2}} \sin ^{2} \theta \cos ^{2} \theta}{1-\beta^{2} \varepsilon_{2} \cos ^{2} \theta}|\zeta|^{2}
$$

where $\zeta$ is given by

$$
\zeta=\frac{\left(\varepsilon_{2}-\varepsilon_{1}\right)\left(1-\beta^{2} \varepsilon_{2}-\beta \sqrt{\varepsilon_{1}-\varepsilon_{2} \sin ^{2} \theta}\right)}{\left(\varepsilon_{1}+\sqrt{\varepsilon_{2}} \sqrt{\varepsilon_{1}-\varepsilon_{2} \sin ^{2} \theta}\right)\left(1-\beta \sqrt{\varepsilon_{1}-\varepsilon_{2} \sin ^{2} \theta}\right)}
$$

$d \omega$ is the bandwidth of the antenna $(d \omega=2 \pi d v) . d \Omega$ is the solid angle subtended by the horn, given by $\Omega=A / d^{2}$ where $d$ is the distance from the horn to the source and $A$ is the area of the horn. $\theta$ is the angle between the beamline and the line to the horn. $\varepsilon_{1}$ and $\varepsilon_{2}$ are the upstream and downstream complex dielectric constants $\left(\varepsilon_{k}=\varepsilon_{k}^{\prime}+i \varepsilon_{k}^{\prime \prime}\right)$, respectively. For these two experiments, the upstream medium is aluminum $\left(\varepsilon_{1}^{\prime} \sim 10\right)$ and the downstream medium is air $\left(\varepsilon_{2}^{\prime}=(1.00035)^{2}\right)$.

The energy is obtained by multiplying the RHS of equation 1 by the bandwidth and solid angle. To convert to power, divide the energy by the typical time of the pulse (about the inverse bandwidth of the antenna). The power delivered to the load, $P_{L}$, is this power multiplied by the horn efficiency, about 0.5 [26].

It is useful to look at the electric field $E$ as well as the power. The Poynting flux $S$ is given by $S=P_{L} / A_{\text {eff }}=E^{2} / 377$, where $A_{\text {eff }}$ is the effective area of the antenna. Therefore $E=\sqrt{377 P_{L} / A_{\text {eff }}}$. We wish to look at the peak electric field, so we multiply $E$ by a factor of $2 \sqrt{2}$, since $E_{\text {peak }}=2 \sqrt{2} E_{R M S}$ for a perfectly triangular envelope.

The finite beam size introduces coherence corrections. The corrected power is given by

$$
P=N_{e}\left(1+N_{e} f_{L} f_{T} \chi\right) P_{0}
$$

where $f_{L}, f_{T}$, and $\chi$ are the longitudinal, transverse and angular form factors, respectively. If the distribution of the electrons is Gaussian, then Shibata et al [39] give the form factors as:

$$
f_{L}=\exp \left[-\left(\pi \sigma_{L} \cos \theta / \lambda\right)^{2}\right]
$$

$$
f_{T}=\exp \left[-\left(\pi \sigma_{T} \sin \theta / \lambda\right)^{2}\right]
$$

$$
\chi=\left(\frac{2 \theta^{2}}{\pi \Omega^{2}} \int_{0}^{\pi / 2 \theta} x[(1-x) K(y)+(1+x) E(y)] e^{-\frac{\theta^{2}}{\Omega^{2}} x^{2}} d x\right)^{2}
$$

where $\sigma_{L}$ and $\sigma_{T}$ are the longitudinal and transverse divergence parameters, $\Omega$ is the angular divergence parameter, and $K(y)$ and $E(y)$ are the complete elliptic integrals of the first and second kinds, where $y=2 \sqrt{x} /(1+x)$ :

$$
\begin{aligned}
& K(y)=\int_{0}^{\pi / 2}\left(1-y^{2} \sin ^{2} \omega\right)^{-1 / 2} d \omega \\
& E(y)=\int_{0}^{\pi / 2}\left(1-y^{2} \sin ^{2} \omega\right)^{1 / 2} d \omega
\end{aligned}
$$

In the perfectly coherent limit, $f_{L}=f_{T}=\chi=1$ and since $N_{e} \gg 1, P=N_{E}^{2} P_{0}$.

To compare the measured power to the calculated power, we take the average of $V^{2}$ over the measured pulse and divide by $50 \Omega$. The measured voltage must be corrected for the attenuation in the circuit: $V_{\text {correct }}=V_{\text {meas }} 10^{\text {atten }(d B) / 20}$.

To get the measured electric field, take the peak electric field $E_{\text {peak }}=2 V_{\text {peak }} / h_{\text {eff }}$ where $h_{\text {eff }}$ is the effective height and $V_{\text {peak }}$ is the measured peak voltage, with a factor of 2 to account for voltage division into a matched load. 


\section{Appendix B: Details of Čerenkov radiation analysis}

Zas, Halzen, and Stanev [41] provided a detailed analysis and simulations of coherent Čerenkov radio emission from high energy showers in ice. A variety of studies since then have refined these initial results and extended them to various scenarios of neutrino flavor and energy [42, 43]. For our analysis, we use the same approach as in our previous SLAC experiment [17], and adapt the parameterizations for the field strength produced by a shower in ice to the following form for salt:

$$
R\left|\mathbf{E}\left(v, R, \theta_{c}\right)\right|=A_{0} f_{d} \kappa\left(\frac{E_{s h}}{1 \mathrm{TeV}}\right) \frac{v}{v_{0}}\left(\frac{1}{1+\left(\frac{v}{v_{1}}\right)^{\delta}}\right)
$$

where $\mathrm{R}$ is the distance from the charged-particle beam in Čerenkov cone direction. $A_{0}$ and $\delta$ are empirically determined coefficients, $A_{0}=2.53 \times 10^{-7}$ and $\delta=1.44$. The factor $f_{d}$ is a scaling factor for the difference of radiation length and density between salt and ice, $f_{d}=0.5$, and $\kappa$ is a factor for antenna near-field effects, approximately 0.5 , with an estimated systematic uncertainty of $\sim 30 \%$ for $\kappa$. Here $E_{s h}$ is the shower energy and is given by $E_{s h}=E_{e} N_{e} t$, where $E_{e}=28.5 \mathrm{GeV}$ is the energy of the electrons in the beam, $N_{e}$ is the number of electrons and $t$ is thickness of radiator in $X_{0}$ (radiation lengths). The parameters $v_{0}=2200 \mathrm{MHz}$, and $v_{1}=3500 \mathrm{MHz}$ determine the decoherence behavior, and are estimated from the parameters for ice, scaled by the ratio of the radiation length in ice and salt, calculated from the empirical formula:

$$
X_{0}=716.4 \frac{\sum_{i} A_{i}}{\sum_{i}\left(Z_{i}\left(Z_{i}+1\right) \ln \frac{287}{\sqrt{Z_{i}}}\right)}\left[\frac{g}{\mathrm{~cm}^{2}}\right]
$$

$Z_{i}$ is atomic number and $A_{i}$ is atomic weight of elements which form the molecule.
[1] F. Halzen, R. J. Protheroe, T. Stanev, \& H. P. Vankov, Phys. Rev. D 4, 342 (1990); J. Wdowczyk \& A. W. Wolfendale, Ap. J. 349, 35 (1990).

[2] K. Greisen, 1966, Phys. Rev. Lett., 16, 748.

[3] G. T. Zatsepin \& V. A. Kuzmin, Pis'ma Zh. Eksp. Teor. Fiz. 4 (1966) 114 [JETP. Lett. 4 (1966) 78].

[4] R. Engel, D. Seckel, and T. Stanev, Phys. Rev. D 64, 093010, (2001).

[5] D. Seckel \& G. Frichter, Proc. 1st Int. Workshop Rad. Detect. High Energy Part. (RADHEP 2000), ed. Saltzberg \& Gorham , (AIP press) (2001).

[6] G. A. Askaryan,1962, JETP 14, 441

[7] G. A. Askaryan,1965, JETP 21, 658

[8] I. Kravchenko et al., Astropart.Phys. 20 (2003) 195-213.

[9] A. Silvestri et al. (2004) astro-ph/0411007; http://www. phys.hawaii.edu/ anita

[10] N. Lehtinen, P. Gorham, A. Jacobson, \& R. Roussel-Duṕre, Phys.Rev. D69 (2004) 013008 ; astro-ph/0309656.

[11] I. M. Zheleznykh, 1988, Proc. Neutrino '88, 528.

[12] R. D. Dagkesamanskii, \& I. M. Zheleznykh, 1989, JETP 50, 233

[13] T. H. Hankins, R. D. Ekers \& J. D. O'Sullivan, 1996, MNRAS 283, 1027.

[14] P.W. Gorham et al., Phys. Rev. Lett. 93 (2004) 041101; astro$\mathrm{ph} / 0310232$.

[15] M. Chiba, T. Kamijo, ). Yasuda, et al., (2004) Phys. Atom. Nuclei 67(11), 2050.

[16] P. Gorham, D. Saltzberg, A. Odian, et al., NIM A490, 476, (2002).

[17] D. Saltzberg, P. Gorham, D. Walz, et al. 2001, Phys. Rev. Lett., 86, 2802.

[18] A. von Hippel, editor, Dielectric Materials and Applications, (1995 ed.), Artech House.

[19] W.R. Nelson, H. Hirayama and D. W. O. Rogers, SLAC-265, Stanford Linear Accelerator Center, 1985; see also http:// www.slac.stanford.edu/egs/.

[20] J. Alvarez-Muñiz, \& E. Zas, 1997, Phys. Lett. B, 411, 218

[21] J. Alvarez-Muñiz, E. Zas Phys.Lett. B434 (1998) 396-406.

[22] P. W. Gorham, D. P. Saltzberg, P. Schoessow, et al., 2000, Phys.
Rev. E. 62, 8590.

[23] D. Williams 2003, unpublished dissertation, UCLA.

[24] G. Varner, P. Gorham, \& J. Cao, (2003), in Proc. of SPIE conf. 4858, Particle Astrophysics Instrumentation, P. Gorham, ed., (SPIE: Bellingham, WA), 285;

[25] G. Varner et al. (2004), http://www.phys.hawaii.edu/ idlab/project_files/salt/docs/GEISER_wu_2.0.pdf

[26] J. D. Kraus, 1988, Antennas, (McGraw-Hill:New York)

[27] R. Gandhi, Nucl.Phys.Proc.Suppl. 91 (2000) 453-461; hep$\mathrm{ph} / 0011176$.

[28] L. A. Anchordoqui, J. L. Feng, H. Goldberg, A. D. Shapere, Phys. Rev. D66 (2002) 103002.

[29] O. E. Kalashev, V. A. Kuzmin, D. V. Semikoz and G. Sigl, Phys. Rev. D 66, 063004 (2002).

[30] R. J. Protheroe \& P. A. Johnson, Astropart. Phys. 4 (1996) 253.

[31] C. Aramo, et al. 2004, Astropart. Phys., in press; astro$\mathrm{ph} / 0407638$.

[32] D. Saltzberg, et al., (2003), Proc. SPIE conf. 4858, Part. Astroph. Instrum., P. Gorham, ed., (SPIE: Bellingham, WA), 191.

[33] M. Ave, N. Busca, A. V. Olinto, A. A. Watson, T. Yamamoto, 2004, astro-ph/0409316.

[34] E. Waxman and J. N. Bahcall, Phys. Rev. D 59, 023002 (1999), hep-ph/9807282.

[35] A. Misaki, et al. Proc. 26th ICRC, Univ. of Utah, HE.3.2.2.24 (1999); hep-ph/9905399.

[36] J. Alvarez-Muñiz, R.A. Vázquez, E. Zas, Phys.Rev. D61 (2000) 023001

[37] J. G. Learned \& S. Pakvasa, Astropart. Phys. 3, 267 (1995).

[38] J. L. Feng \& A. D. Shapere, Phys. Rev. Lett. 88, 021303 (2002); J. Alvarez-Muniz, J. L. Feng, F. Halzen, T. Han, D. Hooper, Phys.Rev. D65 (2002) 124015.

[39] T. Takahashi, Y. Shibata, K. Ishi, et al. 2000, Phys Rev. E 62, 8606.

[40] I. Frank and I. Tamm, Dokl. Akad. Nauk SSSR 14, 109, (1937)

[41] E. Zas, F. Halzen,, \& T. Stanev, 1992, Phys Rev D 45, 362.

[42] J. Alvarez-Muñiz , R.A. Vázquez, E. Zas, Phys. Rev. D62 (2000) 063001.

[43] J. Alvarez-Muñiz, R.A. Vázquez, E. Zas Phys.Rev. D61 (2000) 023001. 